\title{
Modular invariance in finite temperature Casimir effect
}

\author{
Francesco Alessio ${ }^{a}$ and Glenn Barnich ${ }^{b}$ \\ "Dipartimento di Fisica "E. Pancini" and INFN, Università degli studi di Napoli "Federico II", \\ I-80125 Napoli, Italy \\ ${ }^{b}$ Physique Théorique et Mathématique, Université libre de Bruxelles \\ and International Solvay Institutes, \\ Campus Plaine C.P. 231, B-1050 Bruxelles, Belgium \\ E-mail: falessio@na.infn.it, gbarnich@ulb.ac.be
}

ABSTRACT: The temperature inversion symmetry of the partition function of the electromagnetic field in the set-up of the Casimir effect is extended to full modular transformations by turning on a purely imaginary chemical potential for adapted spin angular momentum. The extended partition function is expressed in terms of a real analytic Eisenstein series. These results become transparent after explicitly showing equivalence of the partition functions for Maxwell's theory between perfectly conducting parallel plates and for a massless scalar with periodic boundary conditions.

Keywords: Conformal Field Theory, Discrete Symmetries, Duality in Gauge Field Theories

ARXIV EPRINT: 2007.13334 


\section{Contents}

1 Introduction 1

2 Capacitor partition function $\quad 2$

3 Boundary conditions: $\mathbf{E}$ and $\mathbf{H}$ modes 3

4 The observable $\quad 5$

5 Extended partition function and modular properties $\quad 6$

$\begin{array}{llr}6 & \text { Discussion } & 8\end{array}$

\section{Introduction}

When studying the Casimir effect [1] at finite temperature [2, 3], one cannot fail to be intrigued by the temperature inversion symmetry of the partition function, originally derived using an image-source construction of the Green's function [4]. This result has been re-discussed from various points of view including a derivation in terms of Euclidean path integrals, Jacobi integrals and Epstein zeta functions, while the Casimir energy has been related to the thermodynamic potentials of the system [5-15].

A natural question is then whether one may include another observable in the partition function so as to enhance the temperature inversion symmetry to transformations under the full modular group and complete the parallel to the massless free boson on the torus with momentum operator included. Whereas for two-dimensional conformal field theories on the torus, the central charge has been related to the Casimir energy of a field theory with boundary conditions in one spatial dimension [16-19], we show here how techniques developed in that context can be applied in the original setting of the Casmir effect in order to produce exact results.

In order to address this question, we construct an exact equivalence, at the level of finite temperature partition functions, between Maxwell's theory with perfectly conducting parallel plate boundary conditions on $I_{d} \times \mathbb{R}^{2}$ (or $I_{d} \times \mathbb{T}^{2}$ ), and a free massless scalar on $S_{2 d}^{1} \times \mathbb{R}^{2}$ (or $S_{2 d}^{1} \times \mathbb{T}^{2}$ ). The exact result for the extended partition function then readily follows from that of this scalar field, which has been discussed in detail in [20], and is inline with the analysis of modular invariance in [21].

That the spectrum of electromagnetism with perfectly conducting boundary conditions corresponds to one scalar with Neumann and one scalar with Dirichlet conditions, is discussed implicitly for instance in [22] section 2.4, and explicitly in [23] (see also [24], section 3.2.2). This implies that, when taking perfectly conducting boundary conditions into account, the computation cannot simply be done in terms of 2 polarizations with periodic 
or Dirichlet conditions as in empty space, but one has to base it on $E$ and $H$ modes. It is the $E$ modes that contain the additional $n_{3}=0$ modes because they satisfy Neumann conditions and have thus a cosine expansion in the case of parallel plates. The scalar field theory with periodic boundary conditions on the interval of double the length separating the plates is then constructed out of these $E$ and $H$ modes.

Identifying the correct observable is straightforward in the scalar field formulation. The corresponding expression in terms of electromagnetic fields is somewhat harder to guess directly. The main point here is that the explicit map between the scalar field and the original electromagnetic field is non-local in space. As a consequence, the momentum in this direction in terms of the electromagnetic field, the space integral of $T^{03}$ which is the observable discussed in almost all other investigation of the Casimir effect, does not correspond to scalar field momentum in the $x^{3}$ direction under the map. Rather, as a direct computation using the detailed mode expansion shows, it corresponds to the $x^{3}$-component of a suitably modified version of spin angular momentum of light. This observable is usually not considered in the context of the Casimir effect and that is the reason modular invariance beyond temperature inversion symmetry cannot be discussed either.

Our motivation for studying this question originated from an attempt to understand the contribution of specific degrees of freedom related to non-trivial boundary conditions to partition functions. More precisely, a pair of perfectly conducting plates at constant $x^{3}$ requires Neumann conditions for the third component of the electric field and the vector potential. This gives rise to an additional polarization at zero value of the quantized transverse momentum whose dynamics is governed by a free massless scalar field in $2+1$ dimensions. Its contribution to the partition function scales with the area of the plates and provides the leading correction at low temperature to the zero temperature Casimir result for the free energy $[25,26]$. Just as the contribution of the mode at $n=0$ in the Fourier expansions of a free boson on a torus is essential to achieve modular invariance of the partition function [27, 28], so is the contribution of this lower-dimensional massless scalar field in the current context.

\section{Capacitor partition function}

The partition function for perfectly conducting parallel plates of area $L^{2}$ separated by a distance $d$, such that $L \gg d$, is given by [2-4] (see e.g. [24, 29] for reviews)

$$
\ln Z(t)=\frac{L^{2}}{d^{2}}\left[\frac{\pi^{2} t}{360}-2 t f(1 / t)+\frac{\pi^{2}}{360 t^{3}}\right], \quad t=\frac{\beta}{2 d},
$$

and

$$
f(1 / t)=-\frac{1}{4 \pi^{2}} \sum_{l, m=1}^{\infty} \frac{1}{\left(t^{2} l^{2}+m^{2}\right)^{2}},
$$

or, equivalently, when using a Sommerfeld-Watson transformation,

$$
f(1 / t)=-\sum_{l=1}^{\infty}\left[\frac{1}{16 \pi t^{3} l^{3}} \operatorname{coth}(\pi l t)+\frac{1}{16 t^{2} l^{2} \sinh ^{2}(\pi l t)}\right]+\frac{\pi^{2}}{720 t^{4}} .
$$


The next step $[11,13]$ is to extend the sum over all integers $(l, m)$ except for $(0,0)$ and to recognize the relevant Epstein zeta function,

$$
\mathfrak{Z}\left(2 ; t^{2}, 1\right)=\sum_{(l, m) \in \mathbb{Z}^{2} /(0,0)} \frac{1}{\left(l^{2} t^{2}+m^{2}\right)^{2}},
$$

in order to write the result as

$$
\ln Z(t)=\frac{L^{2} t}{d^{2} 8 \pi^{2}} \mathfrak{Z}\left(2 ; t^{2}, 1\right)
$$

or, in terms of the free energy,

$$
F(t)=-\frac{L^{2}}{d^{3} 16 \pi^{2}} \mathfrak{Z}\left(2 ; t^{2}, 1\right)
$$

The expression for the partition function in (2.1) is in line with the discussion in [4], but differs from the corresponding result for the free energy in [24, 29] by the last term in (2.1), which is absent in the latter references. The reason is that the latter approach includes the subtraction of the full free energy of the black body in empty space, whereas this subtraction is limited to the (divergent) zero temperature part in the former approach. This can be done because the thermal part of the free energy is convergent. The normalization condition chosen here is the one where the standard black body result is recovered at large plate separation (see also e.g. [30, 31] for related discussions).

As pointed out in [13], an advantage of the expression as given in (2.1) is that the inversion symmetry, $f(1 / t)=t^{-4} f(t)$, established in [4], extends to the Epstein zeta function, $\mathfrak{Z}\left(2 ; 1 / t^{2}, 1\right)=t^{4} \mathfrak{Z}\left(2 ; t^{2}, 1\right)$, and thus turns into a symmetry of the full partition function and the free energy,

$$
\ln Z(1 / t)=t^{2} \ln Z(t), \quad F(1 / t)=t^{4} F(t) .
$$

Up to exponentially suppressed terms, the high temperature expansion $t \ll 1$ is

$$
\ln Z(t) \approx \frac{L^{2}}{d^{2}}\left[\frac{\pi^{2}}{360 t^{3}}+\frac{1}{8 \pi} \zeta(3)\right]
$$

where the leading piece is the black body contribution $\frac{V \pi^{2}}{45} \beta^{-3}$ while the sub-leading temperature-independent contribution scales like the area; the low temperature expansion $t \gg 1$ in turn is given by

$$
\ln Z(t) \approx \frac{L^{2}}{d^{2}}\left[\frac{\pi^{2} t}{360}+\frac{1}{8 \pi t^{2}} \zeta(3)\right]
$$

where the second term is due to the lower-dimensional scalar as described above.

\section{Boundary conditions: $\mathrm{E}$ and $\mathrm{H}$ modes}

Let us now provide some details on electromagnetism with Casimir boundary conditions needed for our purpose. We work in radiation gauge $A_{0}=0=\vec{\nabla} \cdot \vec{A}$ and implement from the outset the constraints $\pi^{0}=0=\vec{\nabla} \cdot \vec{\pi}$. Perfectly conducting large parallel plates of sides 
$L$ at $x^{3}=0$ and $x^{3}=d$ with unit normal $\vec{n}$ require $\vec{E} \times \vec{n}=0=\vec{B} \cdot \vec{n}$ on the plates, and periodic boundary conditions in the $x^{a}$ directions. Let $i=1,2,3, a=1,2$,

$$
\begin{aligned}
& k_{a}=\frac{2 \pi}{L} n_{a}, n_{a} \in \mathbb{Z}, \quad k_{3}=\frac{\pi}{d} n_{3}, n_{3} \in \mathbb{N}, \quad k=\sqrt{k_{i} k^{i}}, \quad k_{\perp}=\sqrt{k_{a} k^{a}}, \quad V=d L^{2}, \\
& \psi_{k}^{H}=\sqrt{\frac{2}{V}} e^{i k_{a} x^{a}} \sin k_{3} x^{3}, \quad \psi_{k_{a}, 0}^{E}=\frac{1}{\sqrt{V}} e^{i k_{a} x^{a}}, \quad \psi_{k}^{E}=\sqrt{\frac{2}{V}} e^{i k_{a} x^{a}} \cos k_{3} x^{3}, \quad n_{3} \neq 0 .
\end{aligned}
$$

Following for instance [32] (see also e.g. [22, 23] for closely related discussions), one introduces

$$
\begin{aligned}
\phi^{E} & =\sum_{n_{i}} \frac{1}{\sqrt{2 k} k k_{\perp}}\left[a_{k}^{E} \psi_{k}^{E}+\text { c.c. }\right], \quad \pi^{E}=-i \sum_{n_{i}} \frac{1}{\sqrt{2 k} k_{\perp}}\left[a_{k}^{E} \psi_{k}^{E}-\text { c.c. }\right], \\
\phi^{H} & =\sum_{n_{i}} \frac{1}{\sqrt{2 k} k_{\perp}}\left[a_{k}^{H} \psi_{k}^{H}+\text { c.c. }\right], \quad \pi^{H}=-i \sum_{n_{i}} \frac{\sqrt{k}}{\sqrt{2} k_{\perp}}\left[a_{k}^{H} \psi_{k}^{H}-\text { c.c. }\right],
\end{aligned}
$$

satisfying Neumann, respectively Dirichlet, conditions as well as the Helmholtz equations

$$
\left(\Delta+k^{2}\right) \phi^{\lambda}=0=\left(\Delta+k^{2}\right) \pi^{\lambda},
$$

with $\lambda=(E, H)$. In these terms, the mode expansion of the canonical pair $(\vec{A}, \vec{\pi})$ and the associated electric and magnetic fields $\vec{E}=-\vec{\pi}, \vec{B}=\vec{\nabla} \times \vec{A}$ is given by the sum of $E$ or transverse magnetic modes,

$$
\begin{aligned}
A_{a}^{E} & =\partial_{a} \partial_{3} \phi^{E}, & A_{3}^{E} & =\left(-\Delta+\partial_{3}^{2}\right) \phi^{E}, \\
\pi_{a}^{E} & =\partial_{a} \partial_{3} \pi^{E}, & & \pi_{3}^{E}=\left(-\Delta+\partial_{3}^{2}\right) \pi^{E}, \\
B_{a}^{E} & =\epsilon_{a b} \partial^{b}(-\Delta) \phi^{E}, & B_{3}^{E} & =0,
\end{aligned}
$$

where $\epsilon_{a b}$ is skew-symmetric with $\epsilon_{12}=1$, and $H$ or transverse electric modes,

$$
\begin{aligned}
A_{a}^{H}=\epsilon_{a b} \partial^{b} \phi^{H}, & A_{3}^{H}=0, \\
\pi_{a}^{H}=\epsilon_{a b} \partial^{b} \pi^{H}, & \pi_{3}^{H}=0, \\
B_{a}^{H} & =\partial_{a} \partial_{3} \phi^{H}, \quad B_{3}^{H}=\left(-\Delta+\partial_{3}^{2}\right) \phi^{H},
\end{aligned}
$$

with the understanding that $a_{k_{a}, 0}^{H}=0$. In these variables, the first order electromagnetic action

$$
S=\int \mathrm{d} x^{0}\left[\int_{V} \mathrm{~d}^{3} x \partial_{0} \vec{A} \cdot \vec{\pi}-H\right], \quad H=\int_{V} \mathrm{~d}^{3} x \frac{1}{2}(\vec{E} \cdot \vec{E}+\vec{B} \cdot \vec{B})
$$

is given by

$$
S=\int \mathrm{d} x^{0} \sum_{n_{i}, \lambda}\left[\frac{1}{2 i}\left(\partial_{0} a_{k}^{* \lambda} a_{k}^{\lambda}-a_{k}^{* \lambda} \partial_{0} a_{k}^{\lambda}\right)-k a_{k}^{* \lambda} a_{k}^{\lambda}\right],
$$

with $\lambda=(E, H)$. In particular, Poisson brackets are read off from the kinetic term and oscillators do have the usual time dependence. 
Action (3.7) coincides with the mode expansion of an action for two massless scalar fields, one with Neumann and one with Dirichlet conditions. For later purposes, it is useful to introduce an equivalent formulation in terms of a single free massless scalar field, which satisfies periodic boundary conditions in $x, y$ in intervals of length $L$ and in $x^{3}$ of length $2 d$,

$$
\phi=\frac{1}{\sqrt{V_{P}}} \sum_{n_{i}} \frac{1}{\sqrt{2 k}}\left[a_{k} e^{i k_{j} x^{j}}+\text { c.c. }\right], \quad \pi=\frac{-i}{\sqrt{V_{P}}} \sum_{n_{i}} \sqrt{\frac{k}{2}}\left[a_{k} e^{i k_{j} x^{j}}-\text { c.c. }\right],
$$

where $V_{P}=2 d L^{2}$ with $n_{3} \in \mathbb{Z}$ as well, and

$$
a_{k_{a},-k_{3}}^{E}=a_{k_{a}, k_{3}}^{E}, \quad a_{k_{a},-k_{3}}^{H}=-a_{k_{a}, k_{3}}^{H}, \quad a_{k}=\frac{a_{k}^{E}-i a_{k}^{H}}{\sqrt{2}}, n_{3} \neq 0, \quad a_{k_{a}, 0}=a_{k_{a}, 0}^{E} .
$$

With these definitions, the first order scalar field action

$$
S^{S}=\int \mathrm{d} x^{0}\left[\int_{V_{P}} \mathrm{~d}^{3} x \partial_{0} \phi \pi-H^{S}\right], \quad H^{S}=\int_{V_{P}} \mathrm{~d}^{3} x \frac{1}{2}\left(\pi^{2}+\vec{\nabla} \phi \cdot \vec{\nabla} \phi\right),
$$

agrees with the first order electromagnetic action (3.6) because its expression in terms of modes is given by the r.h.s. of (3.7).

\section{The observable}

In the equivalent scalar field formulation, we will show below that the correct observable to be turned on in order to produce a real part for the modular parameter $\tau$ and to consider full modular transformations is linear momentum in the $x^{3}$ direction,

$$
P_{3}=-\int_{V_{P}} \mathrm{~d}^{3} x \pi \partial_{3} \phi
$$

which, in terms of oscillators, is given by

$$
P_{3}=\sum_{n_{i}} k_{3} a_{k}^{*} a_{k}=\sum_{n_{a}, n_{3}>0} i k_{3}\left(a_{k}^{* H} a_{k}^{E}-a_{k}^{* E} a_{k}^{H}\right) .
$$

The action of this observable in electromagnetic terms can be inferred from

$$
\left\{\phi^{E}, P_{3}\right\}=\left(-\partial_{3}\right)(-\Delta)^{-\frac{1}{2}} \phi^{H}, \quad\left\{\phi^{H}, P_{3}\right\}=\left(-\partial_{3}\right)(-\Delta)^{\frac{1}{2}} \phi^{E},
$$

with similar relations holding for $\pi^{\lambda}$ by using (3.4) and (3.5). On the electromagnetic E and $\mathrm{H}$ vector potentials, electric and magnetic fields, it acts like the curl followed by an application of $\left(-\partial_{3}\right)(-\Delta)^{-\frac{1}{2}}$ and an exchange of $E$ and $H$ :

$$
\begin{aligned}
& \left\{\vec{A}^{E}, P_{3}\right\}=\left(-\partial_{3}\right)(-\Delta)^{-\frac{1}{2}} \vec{B}^{H}, \\
& \left\{\vec{B}^{E}, P_{3}\right\}=\left(-\partial_{3}\right)(-\Delta)^{-\frac{1}{2}} \partial_{0} \vec{E}^{H}, \\
& \left\{\vec{E}^{E}, P_{3}\right\}=\left(-\partial_{3}\right)(-\Delta)^{-\frac{1}{2}}\left(-\partial_{0}\right) \vec{B}^{H},
\end{aligned}
$$


with the transformations of the $H$ fields obtained by exchanging $E$ and $H$ in the above. Similarly, one may show by direct computation that the observable is given by

$$
P_{3}=\int_{V} \mathrm{~d}^{3} x \epsilon^{a b}\left(\sqrt{-\Delta} A_{a}^{E} \pi_{b}^{H}+\sqrt{-\Delta} A_{a}^{H} \pi_{b}^{E}\right) .
$$

Up to multiplication of each term in momentum space by $k$, this observable is the component in the $x^{3}$ direction of spin angular momentum,

$$
\vec{J}=\int_{V} \mathrm{~d}^{3} x \vec{A} \times \vec{\pi},
$$

since one may show that

$$
J_{3}=\int_{V} \mathrm{~d}^{3} x \epsilon^{a b}\left(A_{a}^{E} \pi_{b}^{H}+A_{a}^{H} \pi_{b}^{E}\right) .
$$

\section{$5 \quad$ Extended partition function and modular properties}

For the computation of the extended partition function, the fastet way for our purpose is to follow [33] and to start from the Hamiltonian path integral representation

$$
Z(\beta, \mu)=\operatorname{Tr} e^{-\beta\left(\hat{H}-\mu \hat{P}_{3}\right)}=\int \prod d \phi \prod \frac{d \pi}{2 \pi} e^{-S_{H}^{E}},
$$

where the sum is over periodic phase space path of period $\beta$, and the Euclidean action is

$$
S_{H}^{E}=\int_{0}^{\beta} \mathrm{d} x^{4}\left[\int_{V_{P}} \mathrm{~d}^{3} x\left(-i \partial_{4} \phi \pi\right)+\left(H^{S}-\mu P_{3}\right)\right] .
$$

After integration over the momenta, this leads to

$$
Z(\beta, \mu)=\int \prod d \phi e^{-S_{L}^{E}}
$$

with

$$
S_{L}^{E}=\int_{0}^{\beta} \mathrm{d} x^{4} \int_{V_{P}} \mathrm{~d}^{3} x \frac{1}{2}\left[\left(\partial_{4} \phi+i \mu \partial_{3} \phi\right)^{2}+\partial_{j} \phi \partial^{j} \phi\right] .
$$

Following [34] (see also e.g. [22, 35] for earlier connected work and [36] for a review), the evaluation of this path integral is done by zeta function techniques.

Except for the replacement $\partial_{4} \rightarrow \partial_{4}+i \mu \partial_{3}$, the operator in the action is the Laplacian in 4 dimensions with periodic boundary conditions in all directions. Since the eigenfunctions are $e^{i\left(k_{j} x^{j}+\frac{2 \pi n_{4}}{\beta} x^{4}\right)}$, the eigenvalues are

$$
\lambda_{n_{A}}=(2 \pi)^{2}\left[\sum_{a}\left(\frac{n_{a}}{L}\right)^{2}+\left(\frac{n_{3}}{2 d}\right)^{2}-\left(i \frac{n_{4}}{\beta}-\mu \frac{n_{3}}{2 d}\right)^{2}\right],
$$

where $A=1, \ldots 4$, the zeta function of the capacitor is

$$
\zeta_{C}(s)=\sum_{n_{A}}^{\prime} \lambda_{n_{A}}^{-s}
$$


where the prime means that the term with $n_{A}=0$ is excluded. Note that the scalar field discussed at the end of the introduction corresponds to the modes for which $n_{3}=0$ with $n_{1}, n_{2}, n_{4}$ not all zero.

The next step is to take the limit of large plate size $L$, and hence to turn the sum over the transverse directions turns into integrals. As in two-dimensional conformal field theories on the torus and also in the context of QCD (see e.g. [37-39]), one now uses a purely imaginary chemical potential $\mu=i \nu$ with $\nu$ real. After introducing the complex parameter

$$
\tau=\frac{\nu \beta+i \beta}{2 d},
$$

and doing the integral in polar coordinates, the zeta function becomes

$$
\zeta_{C}(s)=\frac{L^{2} \beta^{2 s-2}}{(2 \pi)^{2 s-1}} \int_{0}^{\infty} \mathrm{d} y \sum_{n_{3}, n_{4}} \frac{y}{\left[y^{2}+\left|\tau n_{3}+n_{4}\right|^{2}\right]^{s}} .
$$

When $n_{3}=0=n_{4}$ the integral is regulated through an infrared cut-off $\epsilon, \int_{\epsilon}^{\infty} \mathrm{d} y y^{-2 s+1}=$ $-\frac{\epsilon^{2-2 s}}{2-2 s}$. This expression together with its derivative both vanish in the limit at $s=0$ in the limit $\epsilon \rightarrow 0$ and can thus be discarded. After performing the integral for the remaining terms, the result may be written in terms of a real analytic Eisenstein series (see e.g. [40] for a recent review),

$$
f_{s}(\tau)=\sum_{(m, n) \in \mathbb{Z}^{2} /(0,0)} \frac{[\mathfrak{I m}(\tau)]^{s}}{|m \tau+n|^{2 s}}
$$

as

$$
\zeta_{C}(s)=-\frac{L^{2}[\mathfrak{I m}(\tau)]^{s-1} f_{s-1}(\tau)}{(2-2 s)(2 \pi)^{2 s-1}(2 d)^{2-2 s}} .
$$

Using the inversion formula,

$$
\pi^{-s} \Gamma(s) f_{s}(\tau)=\pi^{s-1} \Gamma(1-s) f_{1-s}(\tau),
$$

then yields

$$
\zeta_{C}(s)=-\frac{L^{2} \Gamma(2-s)[\mathfrak{I m}(\tau)]^{s-1} f_{2-s}(\tau)}{(1-s) 2^{2 s} \pi^{2}(2 d)^{2-2 s} \Gamma(s-1)}
$$

Since $\Gamma(s-1)^{-1}=-s+O\left(s^{2}\right)$, it follows that $\zeta_{C}(0)=0$, in which case $\ln Z(\tau)=\frac{1}{2} \zeta_{C}^{\prime}(0)$ is explicitly given by

$$
\ln Z(\tau)=\frac{L^{2}}{8 \pi^{2} d^{2}} \frac{f_{2}(\tau)}{\mathfrak{I m}(\tau)} .
$$

The behaviour of the partition function under modular transformations

$$
\tau \rightarrow \tau^{\prime}=\frac{a \tau+b}{c \tau+d}, \quad a d-b c=1,
$$

where $a, b, c, d \in \mathbb{Z}$, then follow directly from modular invariance of $f_{2}(\tau)$,

$$
\ln Z\left(\tau^{\prime}\right)=|c \tau+d|^{2} \ln Z(\tau) .
$$

The previous result (2.5) corresponds to vanishing chemical potential $\nu=0=\mathfrak{R e}(\tau)$ in which case the temperature inversion formula is recovered for $a=0=d, b=-c=1$. 


\section{Discussion}

The main result of the paper is the exact computation in equation (5.13) of the partition function for electromagnetism between two perfectly conducting parallel plates and the operator $P_{3}$ of (4.2), or equivalently (4.5), turned on, in terms of the real analytic Eisenstein series $f_{2}(\tau)$. This result is the analog of the computation of the partition function with momentum operator included, of the free boson on the torus given by

$$
Z_{2}(\tau)=\frac{1}{\sqrt{2 \mathfrak{I m}(\tau)}|\eta(\tau)|^{2}},
$$

which is exactly modular invariant.

More details on a direct derivation in the operator formalism and on an underlying infinite-dimensional symmetry algebra will appear elsewhere. Other formulations making gauge invariance manifest will also be explored. At this stage let us just point out that, after having identified the modular parameter, one may write in the standard way the contribution to the partition function that corresponds to the vacuum energy, i.e., the first term in the r.h.s. of (2.9),

$$
\frac{L^{2} \pi^{2} \beta}{d^{3} 720}=\ln (q \bar{q})^{-c / 24}=\frac{\pi \mathfrak{I m}(\tau) c}{6}, \quad q=e^{2 \pi i \tau},
$$

provided that the central charge of the planar capacitor is taken as

$$
c=\frac{L^{2} \pi}{d^{2} 60} .
$$

\section{Acknowledgments}

The authors are grateful to M. Bonte, G. Giribet, A. Kleinschmidt and P. Niro for comments. This work is supported by the F.R.S.-FNRS Belgium through conventions FRFC PDR T.1025.14 and IISN 4.4503.15.

Open Access. This article is distributed under the terms of the Creative Commons Attribution License (CC-BY 4.0), which permits any use, distribution and reproduction in any medium, provided the original author(s) and source are credited.

\section{References}

[1] H.B. Casimir, On the attraction between two perfectly conducting plates, Kon. Ned. Akad. Wetensch. Proc. 51 (1948) 793 [INSPIRE].

[2] M. Fierz, On the attraction of conducting planes in vacuum, Helv. Phys. Acta 33 (1960) 855 [INSPIRE].

[3] J. Mehra, Temperature correction to the Casimir effect, Physica 37 (1967) 145 [InSPIRE].

[4] L.S. Brown and G.J. Maclay, Vacuum stress between conducting plates: An Image solution, Phys. Rev. 184 (1969) 1272 [InSPIRE].

[5] J.S. Dowker and R. Critchley, Covariant Casimir calculations, J. Phys. A 9 (1976) 535. 
[6] R. Balian and B. Duplantier, Electromagnetic Waves Near Perfect Conductors. II. Casimir Effect, Annals Phys. 112 (1978) 165 [INSPIRE].

[7] S.I. Tadaki and S. Takagi, Casimir effect at finite temperature, Prog. Theor. Phys. 75 (1986) 262 [INSPIRE].

[8] J. Ambjørn and S. Wolfram, Properties of the Vacuum. 1. Mechanical and Thermodynamic, Annals Phys. 147 (1983) 1 [INSPIRE].

[9] J. Ambjørn and S. Wolfram, Properties of the Vacuum. 2. Electrodynamic, Annals Phys. 147 (1983) 33 [INSPIRE].

[10] G. Plunien, B. Müller and W. Greiner, Casimir energy at finite temperature, Physica A $\mathbf{1 4 5}$ (1987) 202 [INSPIRE].

[11] C. Lutken and F. Ravndal, A Symmetry in the Finite Temperature Casimir Effect, J. Phys. A 21 (1988) L793.

[12] L.H. Ford, Spectrum of the Casimir Effect, Phys. Rev. D 38 (1988) 528 [INSPIRE].

[13] F. Ravndal and D. Tollefsen, Temperature inversion symmetry in the Casimir effect, Phys. Rev. D 40 (1989) 4191 [INSPIRE].

[14] V.K. Oikonomou, Study of Temperature Inversion Symmetry for the Twisted Wess-Zumino, J. Phys. A 40 (2007) 5725 [hep-th/0612233] [InSPIRE].

[15] V.K. Oikonomou, Temperature inversion symmetry in gauge-Higgs unification models, Theor. Math. Phys. 159 (2009) 509 [arXiv:0709.1351] [INSPIRE].

[16] A.A. Belavin, A.M. Polyakov and A.B. Zamolodchikov, Infinite Conformal Symmetry in Two-Dimensional Quantum Field Theory, Nucl. Phys. B 241 (1984) 333 [inSPIRE].

[17] J.L. Cardy, Operator Content of Two-Dimensional Conformally Invariant Theories, Nucl. Phys. B 270 (1986) 186 [INSPIRE].

[18] H.W.J. Bloete, J.L. Cardy and M.P. Nightingale, Conformal Invariance, the Central Charge, and Universal Finite Size Amplitudes at Criticality, Phys. Rev. Lett. 56 (1986) 742 [INSPIRE].

[19] I. Affleck, Universal Term in the Free Energy at a Critical Point and the Conformal Anomaly, Phys. Rev. Lett. 56 (1986) 746 [InSPIRE].

[20] A. Cappelli and A. Coste, On the Stress Tensor of Conformal Field Theories in Higher Dimensions, Nucl. Phys. B 314 (1989) 707 [InSPIRE].

[21] E. Shaghoulian, Modular forms and a generalized Cardy formula in higher dimensions, Phys. Rev. D 93 (2016) 126005 [arXiv: 1508.02728] [INSPIRE].

[22] B.S. DeWitt, Quantum Field Theory in Curved Space-Time, Phys. Rept. 19 (1975) 295 [INSPIRE].

[23] D. Deutsch and P. Candelas, Boundary Effects in Quantum Field Theory, Phys. Rev. D 20 (1979) 3063 [INSPIRE].

[24] G. Plunien, B. Müller and W. Greiner, The Casimir Effect, Phys. Rept. 134 (1986) 87 [INSPIRE].

[25] G. Barnich, Black hole entropy from nonproper gauge degrees of freedom: The charged vacuum capacitor, Phys. Rev. D 99 (2019) 026007 [arXiv: 1806.00549] [INSPIRE].

[26] G. Barnich and M. Bonte, Soft degrees of freedom, Gibbons-Hawking contribution and entropy from Casimir effect, arXiv:1912.12698 [INSPIRE]. 
[27] J. Polchinski, Evaluation of the One Loop String Path Integral, Commun. Math. Phys. 104 (1986) 37 [INSPIRE].

[28] C. Itzykson and J.B. Zuber, Two-Dimensional Conformal Invariant Theories on a Torus, Nucl. Phys. B 275 (1986) 580 [inSPIRE].

[29] M. Bordag, G.L. Klimchitskaya, U. Mohideen and V.M. Mostepanenko, Advances in the Casimir effect, Int. Ser. Monogr. Phys. 145 (2009) 1 [INSPIRE].

[30] N.F. Svaiter, The Casimir effect at finite temperature, Nuovo Cim. A 105 (1992) 959 [INSPIRE].

[31] R. Jáuregui, C. Villarreal and S. Hacyan, Finite temperature corrections to the Casimir effect in rectangular cavities with perfectly conducting walls, Annals Phys. 321 (2006) 2156.

[32] F. Borgnis, Elektromagnetische Eingenschwingungen dielektrischer Räume, Annalen Phys. 427 (1939) 359.

[33] J.I. Kapusta, Bose-Einstein Condensation, Spontaneous Symmetry Breaking, and Gauge Theories, Phys. Rev. D 24 (1981) 426 [inSPIRE].

[34] S.W. Hawking, Zeta Function Regularization of Path Integrals in Curved Space-Time, Commun. Math. Phys. 55 (1977) 133 [INSPIRE].

[35] J.S. Dowker and R. Critchley, Effective Lagrangian and Energy Momentum Tensor in de Sitter Space, Phys. Rev. D 13 (1976) 3224 [InSPIRE].

[36] E. Elizalde, S.D. Odintsov, A. Romeo, A.A. Bytsenko and S. Zerbini, Zeta Regularization Techniques with Applications, World Scientific (1994) [DOI].

[37] A. Roberge and N. Weiss, Gauge Theories With Imaginary Chemical Potential and the Phases of $\{Q C D\}$, Nucl. Phys. B 275 (1986) 734 [InSPIRE].

[38] M.G. Alford, A. Kapustin and F. Wilczek, Imaginary chemical potential and finite fermion density on the lattice, Phys. Rev. D 59 (1999) 054502 [hep-lat/9807039] [INSPIRE].

[39] F. Karbstein and M. Thies, How to get from imaginary to real chemical potential, Phys. Rev. D 75 (2007) 025003 [hep-th/0610243] [INSPIRE].

[40] P. Fleig, H.P.A. Gustafsson, A. Kleinschmidt and D. Persson, Eisenstein Series and Automorphic Representations: With Applications in String Theory, Cambridge Studies in Advanced Mathematics, Cambridge University Press (2018) [DOI]. 